\title{
Машиностроение. Металлообработка.
}

УДК.621.914.3

DOI: $10.17277 /$ vestnik.2016.02.pp.303-314

\section{РЕЗЬБОФРЕЗЕРНЫЕ СТАНКИ С ГИДРАВЛИЧЕСКИМИ ВНУТРЕННИМИ (ФОРМООБРАЗУЮЩИМИ) СВЯЗЯМИ НА ОСНОВЕ ШАГОВОГО ГИДРОПРИВОДА}

\author{
В. А. Ванин, А. Н. Колодин, А. В. Солдатов, Д. А. Харин \\ Кафедра «Компьютерные интегрированные системы в машиностроении», \\ ФГБОУ ВО «ТГТУ»; kafedra@mail.gaps.tstu.ru
}

Ключевые слова: внутренние связи; генератор гидравлических импульсов; гидравлические связи; гидравлический шаговый двигатель; гидравлический шаговый привод; резьбофрезерные станки; формообразующие связи.

Аннотация: Рассмотрена возможность применения гидравлических связей с гидравлическими шаговыми двигателями в приводах исполнительных органов станков и построения их по модульному принципу на основе шагового гидропривода. Исследованы механические кинематические цепи, имеющие недостатки: значительную протяженность, сравнительно малую жесткость длинной кинематической цепи и, что более существенно, - жесткость таких цепей не постоянна, так как она определяется в основном жесткостью стыков кинематических пар, которые в процессе движения все время меняются. Точность изготовления звеньев ограничена разумными пределами; повышенное трение, изнашивание приводят к постоянному снижению точности кинематических цепей.

Построены внутренние (формообразующие) кинематические цепи в виде гидравлических связей на основе шагового гидропривода в станках различного технологического назначения с целью повышения точности, снижения металлоемкости, создания рациональной конструкции внутренних (формообразующих) цепей по принципу агрегатно-модульного построения.

\section{Введение}

Резьбофрезерные станки применяются для изготовления резьбы резанием с помощью многолезвийного режущего инструмента, совершающего главное вращательное движение. Заготовка совершает вращательное и поступательное движения подачи, между которыми осуществляется жесткая кинематическая связь.

Резьбофрезерование можно осуществлять двумя способами.

1. Фрезерование кольцевой гребенчатой фрезой с затылованным зубом, ширина которой несколько больше длины нарезаемой части резьбы. Данный метод эффективен при нарезании резьбы на деталях, которые имеют лыски или прорези, при обработке тонкостенных деталей и с центрируемыми резьбами. Оси заготовки и инструмента параллельны и поворот на угол подъема винтовой линии резьбы не производится; при этом заготовка (или инструмент) совершает осевое перемещение соответственно шагу нарезаемой резьбы. 
Осевая подача заготовки осуществляется кулачком настройки шага, значение которого при полном обороте соответствует подъему резьбы заготовки. Это перемещение связано с вращением заготовки. Для каждого значения величины шага резьбы требуется сменный кулачок для настройки.

2. Фрезерование длинных резьб дисковым профильным инструментом дисковой профильной фрезой с затылованным зубом. Ось фрезы устанавливается по отношению к оси заготовки под углом подъема винтовой линии. Движение резания складывается из осевого и вращательного движений инструмента.

Структурные схемы резьбофрезерных станков приведены на рис. 1. Для обоих случаев обработки фрезерованием в станках для обработки резьб и червяков необходимо создать движение скорости резания $\Phi_{v}\left(\mathrm{~B}_{1}\right)$, движение подачи $\Phi_{s}\left(\mathrm{~B}_{2} \Pi_{3}\right)$ по винтовой линии и движение врезания $\mathrm{B}_{p}\left(\Pi_{4}\right)$ на глубину резания. Здесь $\Phi_{v}-$ движение по скорости; $\Phi_{s}$ - движение подачи; $\mathrm{B}_{1}$ и $\mathrm{B}_{2}-$ вращения инструмента и заготовки соответственно; П 3 и $\Pi_{4}-$ продольное и поперечное перемещения инструмента [1].
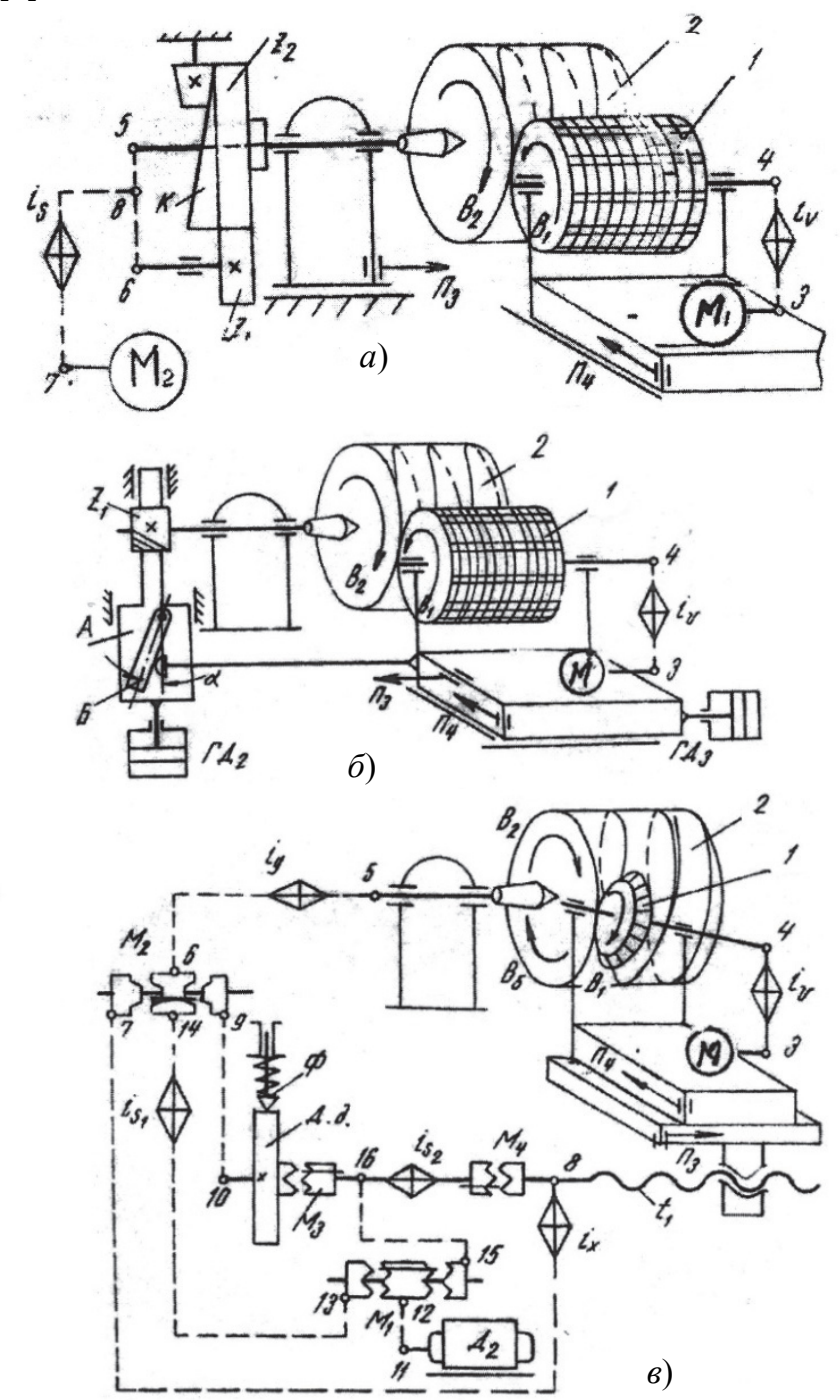

Рис. 1. Варианты кинематической структуры резьбофрезерных станков с использованием в винторезной цепи:

$a$ - кулачкового механизма; $\sigma$ - поворотной линейки; 6 - ходового винта 
Для осуществления в резьбофрезерных станках требуемого формообразующего исполнительного движения между заготовкой и инструментом необходимо создать кинематическую связь между исполнительными звеньями станка и кинематическую связь этих звеньев с источником движения. Кинематические связи между заготовкой и инструментом, необходимые для создания движения формообразования в станках осуществляются с помощью механических кинематических цепей. Они представляют собой совокупность механических звеньев (зубчатых и иных передач, механизмов и устройств, соединенных между собой и предназначенных для выполнения и координации исполнительных движений заготовки и инструмента) как в цепях главного движения и подач, так и во внутренних (формообразующих) цепях станков. Каждая из цепей строится как индивидуализированная конструкция для станков разного габарита и различной точности.

При сложном пространственном расположении исполнительных органов станка (узлов заготовки и инструмента), большом расстоянии между подвижными рабочими органами станка кинематические цепи, составленные из механических звеньев, становятся протяженными, громоздкими, многозвенными, что приводит к усложнению кинематической цепи станка, а также снижению точности функционально связанных линейных или угловых перемещений. Повышение точности внутренних (формообразующих) цепей станков и сохранение ее в процессе эксплуатации возможно реализовать за счет сокращения протяженности кинематических цепей. Необходимо исключить из состава кинематики максимально возможного числа механических звеньев, составляющих цепь (зубчатые и иные передачи, валы, муфты и т.п.), и применить взамен механических гидравлические связи на основе дискретного шагового гидравлического привода. Они обеспечивают высокую точность согласования угловых и линейных перемещений, возможность прямого непосредственного соединения силового исполнительного шагового гидродвигателя с нагрузкой, исключая промежуточные механические передачи, редукторы, коробки подач.

\section{Гидравлические формообразующие связи на основе шагового гидропривода в металлорежущих станках}

Гидравлический шаговый привод, на основе которого осуществляется построение внутренних (формообразующих) цепей станков со сложными движениями формообразования, представляет собой гидромеханическую систему, состоящую из трех автономных функционально и конструктивно завершенных агрегатов (модулей): источника рабочей жидкости (насосная установка), управляющего (коммутирующего) устройства (генератор гидравлических импульсов различного конструктивного исполнения) и силового исполнительного шагового гидродвигателя с механической редукцией шага.

При построении гидравлических формообразующих цепей в качестве силового исполнительного органа используется специальный гидравлический шаговый двигатель (ГШД), выходной вал которого отрабатывает дискретные управляющие сигналы с высокой точностью и большим усилением по мощности. Звеном настройки такой передачи служит генератор гидравлических импульсов, преобразующий энергию рабочей жидкости в гидравлические импульсы давления, которые распределяются в определенной последовательности по рабочим камерам шагового гидродвигателя. Частота вращения и суммарный угол поворота выходного вала ГШД пропорциональны частоте и числу поданных управляющих гидравлических импульсов соответственно. 
Построение внутренних (формообразующих) цепей металлорежущих станков со сложными формообразующими движениями в виде гидравлических связей на основе шагового гидропривода обусловлено тем, что при применении гидравлических связей взамен механических выполняются следующие требования при разработке кинематических цепей:

1) жесткая функциональная кинематическая связь между конечными звеньями цепей, заготовкой и инструментом, с сохранением точного передаточного отношения;

2) возможность регулирования скоростей движения исполнительных органов станка (узлов инструмента и заготовки) и их передаточные отношения в определенном диапазоне;

3) податливость гидравлической связи на основе шагового гидропривода не ниже податливости цепи, состоящей из механических звеньев.

Используя свойства частного регулирования скорости исполнительных органов шагового гидропривода, представляется возможным применять гидравлические связи на основе шагового гидропривода для построения внутренних (формообразующих) цепей, требующих точного взаимосвязанного формообразующего движения заготовки и инструмента. Ликвидируются механические цепи значительной протяженности, которые не обеспечивают жесткую функциональную связь для создания формообразующих движений заготовки и инструмента, как это требуется в резьбофрезерных станках.

Построение внутренних (формообразующих) кинематических цепей на основе синхронной гидравлической связи в виде шагового гидропривода делает возможным не конструировать индивидуально кинематические цепи станков различного технологического назначения каждый раз заново для каждого типа и модели станка, а компоновать их из функционально и конструктивно завершенных агрегатов (модулей), взятых в такой комбинации, при которой обеспечиваются необходимые формообразующие движения, выходная точность станка, рациональная компоновка кинематики станка. Это позволяет значительно упростить кинематическую структуру станка за счет исключения из состава цепей до возможного минимума всех промежуточных звеньев и прямого соединения исполнительного силового шагового двигателя непосредственно с заготовкой и инструментом, без применения промежуточных коробок подач, редукционных передач. Отделение управляющей части системы от силовой для формирования управляющих сигналов без влияния изменения нагрузки на реагирующую часть позволяет создать гидравлическую агрегатную унифицированную систему, из элементов которой возможно скомпоновать внутренние (формообразующие) кинематические цепи станков различного технологического назначения [2 - 5].

\section{Структура внутренних (формообразующих) цепей резьбофрезерных станков на основе шагового гидропривода}

На рисунке 2 представлена структурная схема резьбофрезерного станка с гидравлическими связями в цепях продольной и поперечной подач инструмента с управлением от блока торцовых гидрораспределителей $[6,7]$ для нарезания коротких резьб гребенчатыми фрезами.

Станок включает в себя инструмент (гребенчатую фрезу) 11 , который совершает главное вращательное движение от электродвигателя Д 1 через звено настройки $i_{v}$ и заготовку 10 , осуществляющую вращательное движение круговой подачи от электродвигателя Д2 через звено настройки $i_{s}$. 


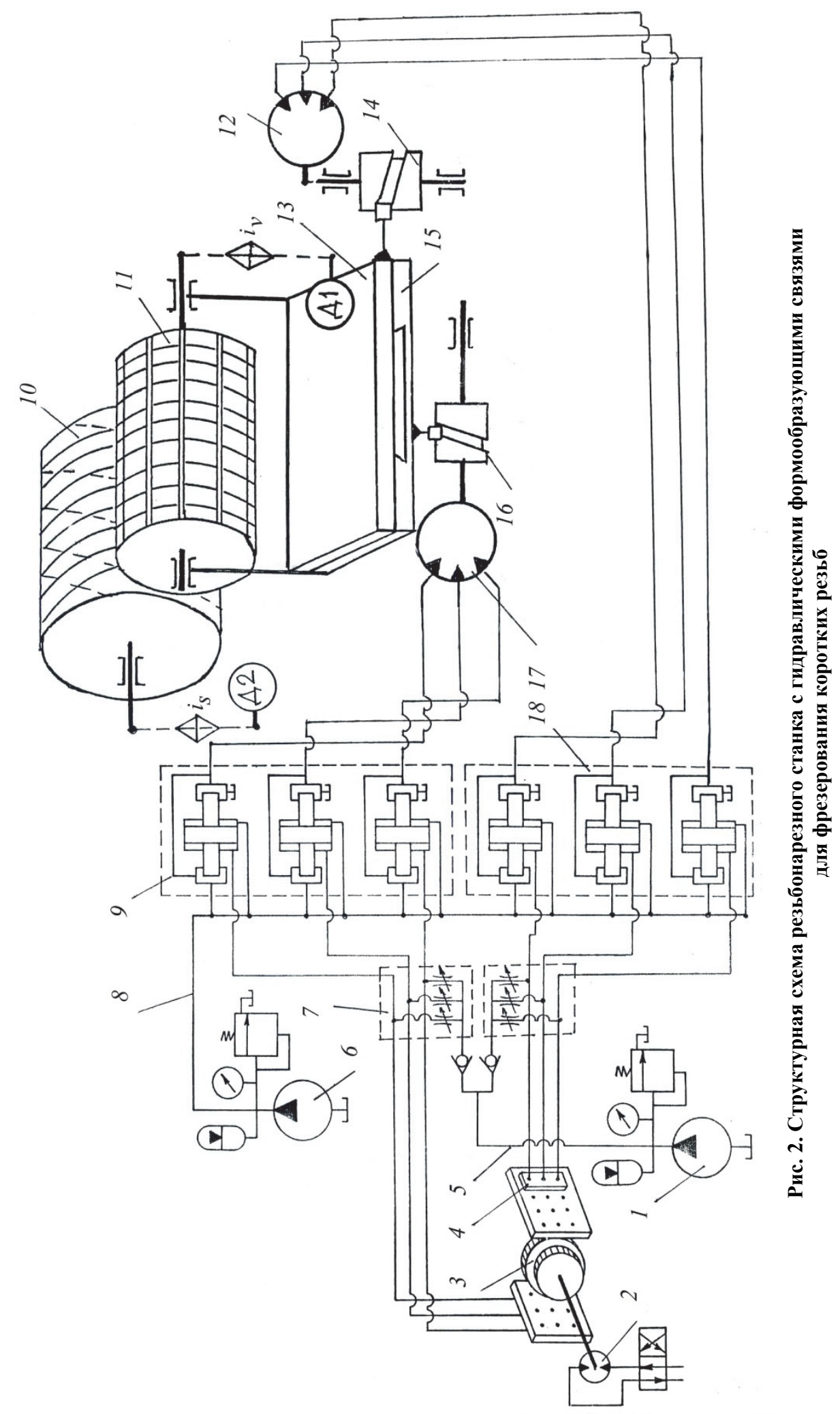

ISSN 0136-5835. Вестник ТГТУ. 2012. Том 22. № 2. Transactions TSTU 
Движение продольной подачи - перемещение фрезы в продольном направлении - осуществляется от шагового гидродвигателя 17, кинематически связанного с барабаном 16 продольной подачи со сменными кулачками. Поперечное перемещение инструмента 11 в радиальном направлении для получения полной глубины фрезерования осуществляется шаговым гидродвигателем 12 , кинематически связанным с барабаном радиальной (поперечной) подачи 14. Управление шаговыми гидродвигателями 17 и 12 продольного и поперечного перемещения фрезерной бабки 13 с инструментом 11 осуществляется от блоков 9 и 18 гидрораспределителей с торцовым распределением рабочей жидкости, выполненных на основе двухкромочного золотника. Настройка цепей продольного и поперечного перемещений фрезерной бабки 13 на требуемое передаточное отношение проводится генератором гидравлических импульсов 3 , представляющим собой набор кодирующих дисков, закрепленных на общей оси и получающих вращение от отдельного гидромотора 2.

Генератор гидравлических импульсов обеспечивает постоянное для данной настройки отношение частот гидравлических импульсов давления, а следовательно, частот вращения выходных валов шаговых гидродвигателей 17 и 12 приводов продольного и поперечного перемещений фрезерной бабки 13 с инструментом 11 . Передаточное отношение между исполнительными движениями гидравлической связи от шаговых гидродвигателей 17 и 12 будет определяться числом гидравлических импульсов, поданных к ним за один оборот блока кодирующих дисков генератора гидравлических импульсов. Изменение передаточного отношения производится перемещением ползушек 4 на корпусе генератора гидравлических импульсов относительно периферии кодирующего диска с разным числом выступов. Коммутация потоков рабочей жидкости осуществляется по силовым каналам в зависимости от того, какая цепь управляющих каналов перекрыта в данный момент выступом вращающегося диска генератора гидравлических импульсов.

Рабочая жидкость для получения управляющих импульсов давления для гидрораспределителей с торцовым распределением поступает от насосной установки 1 через блок постоянных дросселей 7.

Рабочая жидкость от силовой насосной станции 6 поступает на вход блоков дискретных гидрораспределителей 9 и 18 , а затем, в зависимости от положения гидрораспределителей, по одному из силовых каналов передается в рабочие камеры шаговых гидродвигателей 17 и 12 приводов продольной и поперечной подачи фрезерной бабки 13.

На рисунке 3 приведена схема резьбофрезерного станка с гидравлическими формообразующими связями для нарезания цилиндрических винтовых поверхностей переменного шага $[8,9]$ с модифицированным механизмом его приращения.

Станок включает в себя инструмент 8 , представляющий собой дисковую фрезу с профилем, соответствующим профилю нарезаемой винтовой поверхности. Фреза совершает вращательное движение от электродвигателя Д1 через звено настройки $i_{v 1}$ и заготовку, которая совершает вращение (движение круговой подачи) от электродвигателя Д через звено настройки $i_{v}$.

Резьбофрезерный станок включает: кинематическую цепь вращения инструмента (дисковой фрезы); кинематическую цепь вращения шпинделя с заготовкой (движение круговой подачи); кинематическую цепь начального шага, связывающую между собой вращение шпинделя с заготовкой и продольное перемещение

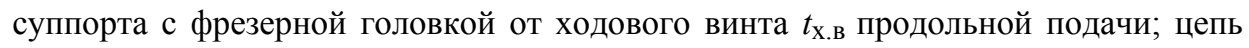
приращения шага винтовой поверхности, связывающую цепь начального шага 


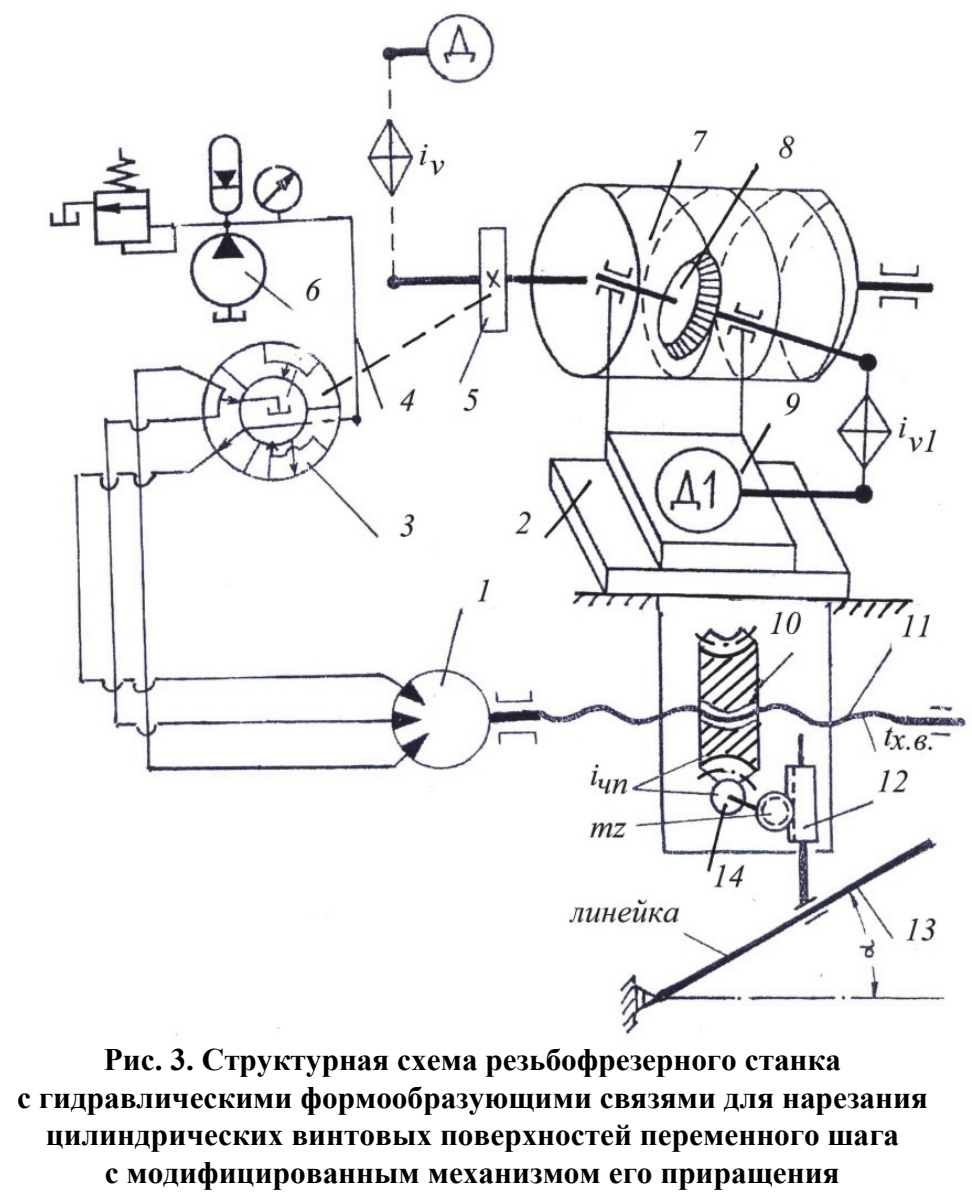

и суммирующий механизм, задающий закон изменения шага, выполненный в виде коррекционной линейки в комбинации с червячной передачей $i_{\text {ч.п. }}$.

Переменный шаг винтовой поверхности образуется в результате сложения двух движений, передаваемых одновременно суппорту станка при его движении: одно - равномерное продольное перемещение по винторезной кинематической цепи от ходового винта, другое - приращение шага винтовой поверхности при включении суммирующего механизма, выполненного в виде коррекционной линейки в комбинации с червячной передачей.

Продольное перемещение суппорта 2 с фрезерной головкой 9 , несущей инструмент 8 (цепь начального шага винтовой поверхности) осуществляется от шагового гидродвигателя 1 , управляемого генератором гидравлических импульсов 3 , золотниковая втулка которого получает вращение от приводного зубчатого колеса 5 , жестко закрепленного на шпинделе заготовки 7.

Дополнительное перемещение продольного суппорта с инструментом, необходимое для получения величины приращения шага винтовой поверхности при продольном перемещении суппорта, осуществляется от коррекционной линейки 13 , установленной под углом к линии центров станка.

При продольном перемещении суппорта 2 с фрезерной головкой 9 одновременно будет перемещаться в поперечном направлении к линии центров станка зубчатая рейка 12 , шарнирно связанная с установленной под углом коррекционной линейкой 13 , и поворачивать на величину $m z$ реечное колесо, кинематически 
связанное с суммирующим механизмом, выполненным в виде червячной передачи на гайке 10 ходового винта 11. Рабочая жидкость к генератору гидравлических импульсов поступает от насосной установки 6 по трубопроводу 4.

На рисунке 4 приведена структурная схема резьбофрезерного станка с гидравлическими формообразующими связями для нарезания цилиндрических винтовых поверхностей переменного шага с модифицированной системой его приращения [10].

Станок включает в себя инструмент 12 , представляющий собой дисковую фрезу, имеющую профиль, соответствующий профилю нарезаемой резьбы и совершающий вращательное движение от электродвигателя Д 1 через звено настройки $i_{v 1}$ и заготовку 11 , которая совершает вращение (движение круговой подачи) от электродвигателя Д через звено настройки $i_{v}$.

Продольное перемещение суппорта 13 с фрезерной головкой 14 , несущей инструмент 12 (цепь начального шага винтовой поверхности) осуществляется от шагового гидродвигателя 4, который управляется генератором гидравлических импульсов 10 , золотниковая втулка с расчетным числом рабочих щелей которого получает вращение от приводного зубчатого колеса 7 , жестко закрепленного на шпинделе заготовки 11.

Переменный шаг винта образуется в результате того, что суппорту сообщается одновременно два движения: одно - с постоянной скоростью от ходового винта 15 , другое - с переменной скоростью за счет дополнительного поворота ходового винта по дополнительной кинематической цепи.

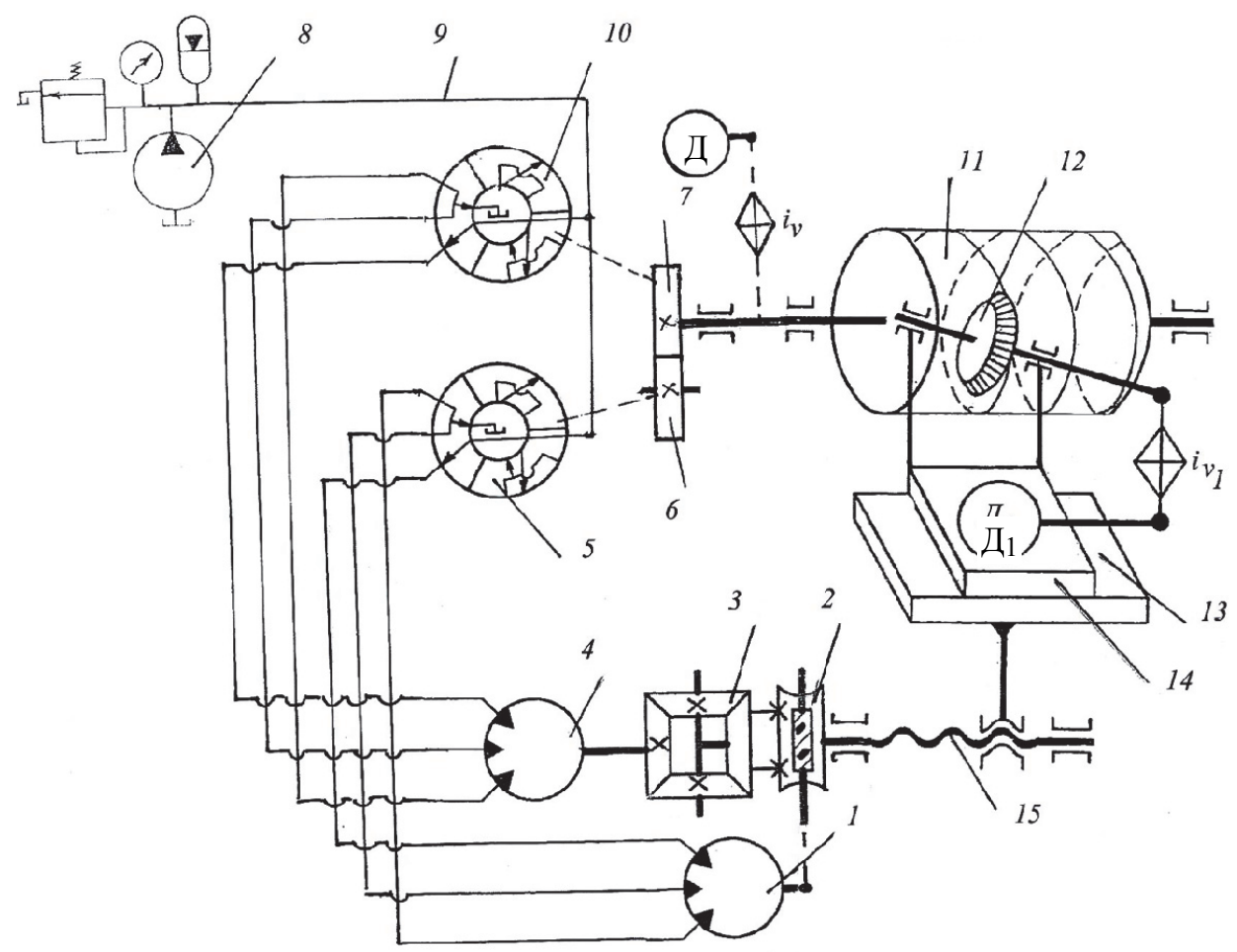

Рис. 4. Структурная схема резьбофрезерного станка с гидравлическими формообразующими связями для нарезания цилиндрических винтовых поверхностей переменного шага 
Дополнительное перемещение инструмента, соответствующее величине приращения шага винтовой линии, осуществляется от шагового гидродвигателя 1 , кинематически связанного с суппортом 13 червячной передачей 2 с суммирующим механизмом 3 в виде дифференциала с коническими колесами. Шаговый гидродвигатель управляется генератором гидравлических импульсов 5, золотниковая втулка которого получает вращение от шпинделя заготовки через цилиндрические зубчатые колеса 7 и 6. Рабочая жидкость к генератору гидравлических импульсов поступает от насосной установки 8 по трубопроводу 9.

\section{Заключение}

Применение гидравлического шагового привода при построении внутренних (формообразующих) кинематических цепей в виде гидравлических связей взамен механических дает возможность решить следующие задачи при проектировании станков.

1. Обеспечить конструктивную однородность внутренних кинематических цепей для всех типов станков различного технологического назначения, в которых при работе требуется осуществить жесткую функциональную связь между инструментом и заготовкой. Построение рациональной компоновки сложных формообразующих цепей выполняется из ограниченной номенклатуры составных унифицированных агрегатов (модулей), имеющих функциональную и конструктивную завершенность. Использование ограниченного числа деталей и узлов индивидуального проектирования и изготовления обеспечивает построение внутренних цепей различного функционального назначения, выполняющих технологические и компоновочные условия по точности, числу формообразующих движений, металлоемкости для конкретной технологической задачи (цепей деления, обката, винторезных, дифференциальных, затылования, и т.п.) в станках различного технологического назначения.

2. Расширить возможность применения шагового гидропривода для выполнения сложных взаимосвязанных формообразующих движений в станках, особенно при сложном пространственном расположении рабочих органов станка, при большом числе промежуточных звеньев в цепи, значительном расстоянии между подвижными рабочими органами, когда механические кинематические цепи становятся сложными и громоздкими, приводящими к усложнению конструкции станка и снижению точности функционально связанных перемещений.

3. Позволить не конструировать внутренние кинематические цепи станков различного технологического назначения и равных типоразмеров одного назначения каждый раз заново. Компоновать их из функционально и конструктивно завершенных агрегатов (модулей), взятых в таком сочетании, чтобы обеспечить необходимые формообразующие движения, рациональную компоновку станка, точность цепей.

4. Уменьшить металлоемкость и массу станка за счет исключения из состава механической цепи до возможного минимума число промежуточных звеньев (зубчатых и иных передач и т.п.) при замене ее гидравлической связью. Это приводит к упрощению кинематики станка, уменьшению габаритных размеров узлов, несущих инструмент и заготовку, повышению жесткости их корпусов благодаря устранению окон и полостей в них для размещения кинематических передач, также созданию более рациональной компоновки с предельно сближенными узлами инструмента и заготовки при сложном пространственном расположении рабочих органов станка. 


\title{
Список литературь
}

1. Федотенок, Л. А. Кинематическая структура металлорежущих станков / Л. А. Федотенок. - М. : Машиностроение, 1970. - 403 с.

2. Колодин, А. Н. Затыловочные станки с гидравлическими формообразующими связями : монография / А.Н. Колодин, В. А. Ванин, А. А. Родина. Deutschland : LAP Lambert Academic Publishing, 2015. - 169 c.

3. Ванин, В. А. Станки с гидравлическими внутренними (формообразующими) связями на основе шагового гидропривода для обработки винтовых поверхностей / В. А. Ванин, А. Н. Колодин // Справочник. Инженер. журн. с прил. 2012. - № 7 (184). - C. 30 - 35.

4. Ванин, В. А. Металлорежущие станки с гидравлическими связями на основе шагового гидропривода во внутренних (формообразующих) цепях / В. А. Ванин [и др.] // Вестн. Тамб. гос. техн. ун-та. - 2013. - Т. 19, № 1. - С. 167 - 176.

5. Применение шагового гидропривода для построения формообразующих цепей металлорежущих станков со сложными движениями формообразования / В. А. Ванин [и др.] // Вопр. соврем. науки и практики. Университет им. В. И. Вернадского. - 2014. - № 1(50). - С. 250 - 259.

6. Ванин, В. А. Применение гидравлического шагового привода для построения внутренних цепей металлорежущих станков / В. А. Ванин, А. Н. Колодин // Вестн. машиностроения. - 2010. - № 5. - С. $19-23$.

7. Ванин, В. А. Агрегатно-модульное построение внутренних (формообразующих) кинематических цепей металлорежущих станков на основе шагового гидропривода / В. А. Ванин, А. Н. Колодин // СТИН. - 2010. - № 9. - С. 2 - 6.

8. Ванин, В. А. Агрегатно-модульное построение внутренних (формообразующих) кинематических цепей металлорежущих станков на основе шагового гидропривода / В. А. Ванин, А. Н. Колодин // СТИН. - 2010 - № 10. - С. 2 - 5.

9. Vanin, V. A. Modular Design Based on Hydraulic Step Drives for Internal Kinematic Chains in Metal-Cutting Machines / V. A. Vanin, A. N. Kolodin // Russian Engineering Research. - 2010. - Vol. 30, No. 12. - P. 1248 - 1251.

10. Vanin, V. A. Application of Hydraulic Step Drives in Metal-Cutting Machine Tools / V. A. Vanin, A. N. Kolodin // Russian Engineering Research. - 2010. - Vol. 30, No. 5. - P. $446-450$.

\section{Thread-Milling Machine Tools with Hydraulic (Formative) Connections on the Base of Stepping Hydraulic Drive}

\author{
V. A. Vanin, A. N. Kolodin, A. V. Soldatov, D. A. Kharin \\ Department of Computer Integrated System in Mechanical Engineering, TSTU; \\ kafedra@mail.gaps.tstu.ru
}

Keywords: forms-formative connection; hydraulic hookup; hydraulic pulse generator; hydraulic stepping motor; the internal hookup; stepping hydraulic drive; thread milling machine tools.

Abstract: This article discusses the possibility of using hydraulic connections with stepper motors in the actuators of machines, the movements of which are strictly coordinated with each other in a certain kinematic dependence; we also consider the possibility of building such connections on a modular principle using a hydraulic stepper. 
Composed of mechanical connections, kinematic chains are designed and constructed for each configuration of the machine used for the same purpose, but having different dimensions. In addition, mechanical kinematic chains have significant drawbacks: a considerable length and a relatively low stiffness. Apart from this, the stiffness of these chains is not constant because it is determined mainly by the stiffness of joints of kinematic pairs, which change all the time due to movement; in addition, the manufacturing accuracy of these connections is restricted within reasonable limits. Excessive friction and wear lead to a steady decrease in the accuracy of kinematic chains.

We also considered the possibility of building internal (formative) kinematic chains in the form of hydraulic connections using a stepping motor in machines of various technological purposes to increase the accuracy, reduce metal consumption, create a rational design of internal (formative) chains using a modular principle.

\section{References}

1. Fedotenok A.A. Kinematicheskaya struktura metallorezhushchikh stankov [Kinematic structure of machine tools], Moscow: Mashinostroenie, 1970, 403 p. (In Russ.)

2. Kolodin A.N., Vanin V.A., Rodina A.A. Zatylovochnye stanki $s$ gidravlicheskimi formoobrazuyushchimi svyazyami [Backing machines with hydraulic formative relationships], Deutschland: LAP Lambert Academic Publishing, 2015, 169 p. (In Russ.)

3. Vanin V.A., Kolodin A.N. [Machines with hydraulic internal (formative) linkages based on hydraulic stepper for processing screw surfaces], Spravochnik. Inzhenernyi zhurnal [Handbook. An Engineering journal with appendix], 2012, no. 7 (184), pp. 30-35. (In Russ., abstract in Eng.)

4. Vanin V.A., Kolodin A.N., Do M.Z., Damap M.M. [Metal-cutting machines with hydraulic connections on the basis of hydraulic stepper drive in internal (shaping) chains], Vestnik Tambovskogo gosudarstvennogo tekhnicheskogo universiteta [Transactions of the Tambov State Technical University], 2013, vol. 19, no. 1, pp. 167-176. (In Russ., abstract in Eng.)

5. Vanin V.A., Kolodin A.N., Averin A.S., Khramova N.A. [Application of the hydraulic drive for the construction of stepper shaping circuits of machine tools with complex movements shaping], Voprosy sovremennoi nauki i praktiki. Universitet im. V.I. Vernadskogo [Problems of Contemporary Science and Practice. Vernadsky University], 2014, no. 1(50), pp. 250-259. (In Russ., abstract in Eng.)

6. Vanin V.A., Kolodin A.N. Application of hydraulic step drives in metal-cutting machine tools, Russian engineering research, 2010, vol. 30, no. 5, pp. 446-450.

7. Vanin V.A., Kolodin A. N. [Aggregate-modular construction of internal (formative) kinematic chains of machine tools based on hydraulic stepper], STIN, 2010, no. 9, pp. 2-6. (In Russ., abstract in Eng.)

8. Vanin V.A., Kolodin A. N. [Aggregate-modular construction of internal (formative) kinematic chains of machine tools based on hydraulic stepper], STIN, 2010, no. 10, pp. 2-5. (In Russ., abstract in Eng.)

9. Vanin V.A., Kolodin A. N. Modular design based on hydraulic step drives for internal kinematic chains in metal-cutting machines, Russian Engineering Research, 2010, vol. 30, no. 12, pp. 1248-1251.

10. Vanin V.A., Kolodin A. N. Application of hydraulic step drives in metalcutting machine tools, Russian Engineering Research, 2010, vol. 30, no. 5, pp 446-450. 


\section{Gewindefräsmaschinen mit den hydraulischen inneren (formbildenden) Beziehungen aufgrund des Schritthydroantriebes}

Zusammenfassung: Es ist die Möglichkeit der Anwendung der hydraulischen Beziehungen mit den hydraulischen Schrittmotoren in den Antrieben der Stellteilen der Werkbänke, deren Bewegungen untereinander in einer bestimmten kinematischen Abhängigkeit koordiniert sind, und der Aufbau solcher Beziehungen nach dem modularen Prinzip aufgrund des Schritthydroantriebes betrachtet. Es klärt sich damit, dass die kinematischen Ketten, die aus den mechanischen Gliedern gebildet sind, individuell unter jede Zusammenstellung der Werkbank der selben Zweckbestimmung, aber der verschiedenen Abmessung projektiert und gebaut werden. Außerdem haben die mechanischen kinematischen Ketten die wesentlichen Mängel: die bedeutende Ausdehnung, die verhältnismäßig kleine Härte der langen kinematischen Kette und, was wesentlicher ist - ist die Härte solcher Ketten nicht ständig, da sie hauptsächlich von der Härte der Grenzen der kinematischen Paare bestimmt wird, die sich im Laufe der Bewegung die ganze Zeit ändern und, außerdem ist die Genauigkeit der Herstellung der Glieder von den vernünftigen Grenzen beschränkt; die erhöhte Reibung, der Verschleiß bringen zur ständigen Senkung der Genauigkeit der kinematischen Ketten.

Es ist die Möglichkeit des Aufbaues der inneren (formbildenden) kinematischen Ketten in Form der hydraulischen Beziehungen aufgrund des Schritthydroantriebes in den Werkbänken der verschiedenen technologischen Zweckbestimmung zwecks der Erhöhung der Genauigkeit, der Senkung des Metallanteils, der Schaffung der Rationalkonstruktion der (formbildenden) Kette mit der Benutzung des Prinzipes des Aggregatmodulaufbaues betrachtet.

\section{Machines à fraiser avec des liens hydrauliques internes (formants) à la base de la commande hydraulique pas à pas}

Résumé: Est considérée la possibilité de l'application des liens hydrauliques avec le moteur hydraulique pas à pas dans les commandes des organes exécutifs des machines-outils dont les mouvements sont strictement coordonnés entre eux dans une certaine dépendance cinématique.

Cela s'explique par le fait que les chaînes cinématiques composées des liens mécaniques sont conçues et construites individuellement pour chaque composition de la machine, ont la même destination mais sont de différents gabarits.

En outre, les chaînes cinétiques mécaniques ont des inconvénients importants: grande étendue; rigidité relativement faible le long de la chaîne cinématique; rigidité qui n'est pas constante car elle est déterminée principalement par la rigidité des joints des couples cinématique.

Est examinée la possibilité de la construction des liens hydrauliques internes (formants) à la base de la commande hydraulique pas à pas dans les machines de la destination technologique différente.

Авторы: Ванин Василий Агафонович - доктор технических наук, профессор кафедры «Компьютерно-интегрированые системы в машиностроении»; Колодин Андрей Николаевич - ассистент кафедры «Компьютерно-интегрированые системы в машиностроении»; Солдатов Андрей Владиславович - студент; Харин Дмитрий Александрович - студент, ФГБОУ ВО «ТГТУ».

Рецензент: Мордасов Денис Михайлович - доктор технических наук, профессор, заведующий кафедрой «Материалы и технология», ФГБОУ ВО «ТГТУ». 